\title{
Fuzzy set theoretic approach to fault tree analysis
}

\author{
Sanjay Kumar Tyagi ${ }^{*}$, D. Pandey ${ }^{2}$ and Reena Tyagi ${ }^{3}$ \\ ${ }^{1}$ Department of Mathematics, Amity School of Engineering and Technology, Amity University Uttar Pradesh, Noida-201301, INDIA \\ ${ }^{2}$ Department of Mathematics, C.C.S. University, Meerut-250004, INDIA \\ ${ }^{3}$ Department of Electronics and Communications, R.D. Engineering College, Ghaziabad-201206, INDIA \\ *Corresponding author: email: sanjay_tyagi94@rediffmail.com, Tel: +91-9871015864
}

\begin{abstract}
Research in conventional fault tree analysis (FTA) is based mainly on failure probability of basic events, which uses classical probability distributions for the failure probability of basic events. In the present paper the probabilistic consideration of basic events is replaced by possibilities, thereby leading to fuzzy fault tree analysis. Triangular and trapezoidal fuzzy numbers are used to represent the failure possibility of basic events. Since a system may have to go through different operating conditions during the design or testing phase. Thus the failure possibility of a basic event will be assigned more than one fuzzy numbers by different experts under various operating conditions. It is also well established that the selection of a fuzzy number to represent a basic event is vital in fault tree analysis. Here we developed an algorithm to find a single fuzzy number for a basic event, wherein more than one fuzzy number is assigned to that particular event. Using this algorithm, we obtain a single fuzzy number, having least variance from all fuzzy numbers assigned to the concerned event. The adequate and appropriate means and procedure for the detection of basic events having key role in the occurrence of top event in system analysis become essential. Here we have put forward an approach to rank the basic events in accordance with their importance in the occurrence of top event. This approach can be widely used to improve the reliability and to reduce the operating cost of a system. The proposed techniques are discussed and illustrated by taking an example of a nuclear power plant.
\end{abstract}

68M15 (Reliability, testing and fault tolerance)

Keywords: Fault tree, Triangular and Trapezoidal fuzzy number, Fuzzy importance, Ranking of fuzzy numbers

\section{Introduction}

Fault tree analysis (FTA) seems to be a very effective tool to predict probability of hazard, resulting from sequences and combinations of faults and failure events. A fault tree is a logical and graphical description of various combinations of failure events. To depict a fault tree, first we determine the hazards and then look for the events causing this hazard. In conventional FTA based on a probabilistic approach the basic events are represented by the probabilities (crisp numbers). However for the system like nuclear power plants, space shuttles, clinical appliances etc., wherein available data are insufficient for statistical inference (Jackson et al., 1981), it is often very difficult to estimate precise failure rates of the basic events. For such systems it is therefore unrealistic to assume a crisp number (classical) for different basic events. Zadeh (1965) suggested a paradigm shift from a theory of total denial and affirmation to a theory of grading to give new concept of fuzzy set. Tanaka and Singer (1983, 1990) then used fuzzy set theory to replace crisp numbers by fuzzy numbers for better estimation of possibility of top event in FTA. Suresh et al (1996) used a method based on $\alpha$-cuts to deal with FTA, treating the failure possibility as triangular and trapezoidal fuzzy numbers.

Accurate failure data is a crucial requirement for reliability assessment. In many situations, where human judgment, evaluation and decision-making are important, failure data may not be corrected accurately. It might sometime require linguistic terms to express data value (Pandey and Tyagi, 2007). But if more than one fuzzy number is assigned to a particular event then random selection of any of these fuzzy numbers to determine the failure possibility of this event is not realistic. This work proposes a method to obtain a single fuzzy number having least variance with the fuzzy numbers assigned to that particular event. 
In FTA the concept of importance may be used to make some vital modifications in the designing of system. Furuta and Shiraishi (1984) proposed the concept of fuzzy importance using max-min fuzzy operator and fuzzy integral. Pan and Tau (1988) developed a model for computing the importance measure of basic events using variance importance measure. Monte-Carlo simulation is generally used in the determination of variance importance measure even though computing process is time taking in this method. Thus for a very complex system having large number of components, the whole procedure has to be repeated again and again, thus not suitable for the fuzzy approach. Suresh et al. (1996) proposed another method to evaluate an importance measure called fuzzy importance measure (FIM).For effective evaluation of the importance index of each basic events, we have introduced a comparatively easier method to calculate fuzzy importance index (FII), based on ranking of fuzzy numbers and Hamming distance. The proposed methods are demonstrated by taking an example of nuclear power plant.

\section{Fuzzy numbers and arithmetic operations}

(A) Fuzzy numbers: A fuzzy set $\tilde{A}$ defined on $R$ must possess the following properties to qualify as a fuzzy number:

(a) $\quad \mu_{\tilde{A}}(x)=0$ for all $x \in(-\infty, \mathrm{c}] \cup[d, \infty)$, where $c<d$.

(b) $\quad \mu_{\tilde{A}}(x)$ is strictly increasing on $[\mathrm{c}, a]$ and strictly decreasing on $[b, d]$ for $c \leq a \leq b \leq d$.

(c) $\mu_{\tilde{A}}(x)=1$ for all $x \in[a, b]$ provided $a \leq b$.

(i) Triangular fuzzy numbers: A fuzzy number $\tilde{A}$ is termed as triangular fuzzy number if the membership function of fuzzy number $A$ is defined by the following expression:

$$
\mu_{\tilde{A}}(x)= \begin{cases}0 & \text { if } x \leq a_{1} \text { or } x \geq a_{2} \\ \frac{x-a_{1}}{a_{2}-a_{1}} & \text { if } a_{1} \leq x \leq a_{2} \\ \frac{a_{3}-x}{a_{3}-a_{2}} & \text { if } a_{2} \leq x \leq a_{3}, \quad x, a_{1}, a_{2}, a_{3} \in R\end{cases}
$$

A triplet $\left(a_{1}, a_{2}, a_{3}\right)$ may be used to denote each triangular fuzzy number defined above.

(ii) Trapezoidal fuzzy numbers: A trapezoidal fuzzy number $\tilde{A}$ denoted by a quadruple $\left(a_{1}, a_{2}, a_{3}, a_{4}\right)$ can be defined as follows:

$$
\mu_{\tilde{A}}(x)= \begin{cases}0 & \text { if } x \leq a_{1} \text { or } x \geq a_{3} \\ \frac{x-a_{1}}{a_{2}-a_{1}} & \text { if } a_{1} \leq x \leq a_{2} \\ 1 & \text { if } a_{2} \leq x \leq a_{3} \\ \frac{a_{4}-x}{a_{4}-a_{3}} & \text { if } a_{3} \leq x \leq a_{4}, \quad x, a_{1}, a_{2}, a_{3}, a_{4} \in R\end{cases}
$$

(B) Operations on triangular and trapezoidal fuzzy numbers: The addition of triangular fuzzy number $\tilde{A}=\left(a_{1}, a_{2}, a_{3}\right)$ and $\tilde{B}=\left(b_{1}, b_{2}, b_{3}\right)$ is defined as:

$$
\tilde{A}+\tilde{B}=\left(a_{1}+b_{1}, a_{2}+b_{2}, a_{3}+b_{3}\right)
$$

Thus the addition of two triangular fuzzy numbers is again a triangular fuzzy number.

Similarly subtraction of two triangular fuzzy numbers is also a triangular fuzzy number and it can be given by the following expressions:

$$
\tilde{A}-\tilde{B}=\left(a_{1}-b_{1}, a_{2}-b_{2}, a_{3}-b_{3}\right)
$$

The multiplication of two fuzzy numbers $\tilde{A}=\left(a_{1}, a_{2}, a_{3}\right)$ and $\tilde{B}=\left(b_{1}, b_{2}, b_{3}\right)$ denoted as $A^{*} B$ can be defined as:

$$
\mu_{\tilde{A} * \tilde{B}}(x)=\left\{\begin{array}{cl}
-D_{1}+\left[D_{1}^{2}+(x-P) / T_{1}\right]^{1 / 2} & P \leq x \leq Q \\
-D_{1}-\left[D_{2}^{2}+(x-R) / U_{1}\right]^{1 / 2} & Q \leq x \leq R, \\
0 & \text { otherwise }
\end{array}\right.
$$

where $T_{1}=\left(a_{2}-a_{1}\right)\left(b_{2}-b_{1}\right), T_{2}=a_{1}\left(a_{2}-a_{1}\right)+b_{2}\left(b_{2}-b_{1}\right), U_{1}=\left(a_{2}-a_{1}\right)\left(b_{2}-b_{1}\right), U_{2}=b_{3}\left(a_{2}-a_{1}\right)+a_{3}\left(b_{2}-b_{1}\right), D_{1}=\frac{T_{2}}{2 T_{1}}, D_{2}=-\frac{U_{2}}{2 U_{1}}, P=a_{1} b_{1}$, $Q=a_{2} b_{2}, R=a_{3} b_{3}$. 
It is evident that the resulting fuzzy number $\tilde{A} * \tilde{B}$ is not a triangular fuzzy number. But in most of the cases, computation with resulting fuzzy numbers becomes very tedious. Thus it is necessary to avoid the second and higher degree terms to make them computationally easy and therefore the product of two fuzzy numbers is reduced to a triangular fuzzy number $(P, Q, R)$ or $\left(a_{1} b_{1}\right.$, $\left.a_{2} b_{2,} a_{3} b_{3}\right)$.

With a similar approach one can define the algebraic operations on trapezoidal fuzzy numbers. Thus the addition, subtraction and multiplication of two trapezoidal fuzzy numbers $\tilde{A}=\left(a_{1}, a_{2}, a_{3}, a_{4}\right)$ and $\tilde{B}=\left(b_{1}, b_{2}, b_{3}, b_{4}\right)$ are again represented by the trapezoidal fuzzy numbers $\left(a_{1}+b_{1}, a_{2}+b_{2}, a_{3}+b_{3}, a_{4}+b_{4}\right),\left(a_{1}-b_{1}, a_{2}-b_{2}, a_{3}-b_{3}, a_{4}-b_{4}\right)$ and $\left(a_{1} b_{1}, a_{2} b_{2}, a_{3} b_{3}, a_{4} b_{4}\right)$ respectively.

\section{Fuzzy operators}

Using algebraic operations on fuzzy numbers (triangular or trapezoidal) we can obtain fuzzy operators FNOT, ANDF and ORF corresponding to Boolean operators NOT, AND and OR respectively as follows.

(i) If a fuzzy event ' $i$ ' is represented by a possibility function $\tilde{p}_{i}$, the generalized Boolean operator NOT to be denoted by FNOT and defined as:

$$
\begin{aligned}
& \text { FNOT } p_{i}=1-p_{i}=1-\left(a_{i 1}, a_{i 2}, a_{i 3}\right)=\left(1-a_{i 3}, 1-a_{i 2}, 1-a_{i 1}\right) \text { (for triangular fuzzy numbers) } \\
& \text { FNOT } p_{i}=1-p_{i}=1-\left(a_{i 1}, a_{i 2}, a_{i 3}, a_{i 4}\right)=\left(1-a_{i 4}, 1-a_{i 3}, 1-a_{i 2}, 1-a_{i 1}\right) \text { (for trapezoidal fuzzy numbers) }
\end{aligned}
$$

(ii) If $\tilde{p}_{1}, \tilde{p}_{2}, \ldots \ldots \ldots . \tilde{p}_{n}$ are the possibility functions of $n$ basic events and $\tilde{p}_{y}$ be the same for resulting event. Then the fuzzy operators $A N D F$ and $O R F$ are defined in the following manner:

(a) $\quad \tilde{p}_{y}=\operatorname{ANDF}\left(\tilde{p}_{1}, \tilde{p}_{2}, \ldots \ldots \ldots \ldots \ldots \ldots \tilde{p}_{n}\right)=\prod_{i=1}^{n} \tilde{p}_{i}$, where $\Pi$ denotes the fuzzy multiplication and $\tilde{p}_{y}$ be the possibility of resulting event.

Let $\tilde{p}_{i}$ 's are represented by triangular fuzzy numbers $\left(a_{i 1}, a_{i 2}, a_{i 3}\right), i=1,2 \ldots n$, then

$$
\tilde{p}_{y}=\operatorname{ANDF}\left(\tilde{p}_{1}, \tilde{p}_{2}, \ldots \ldots \ldots \ldots \ldots . . . . . \tilde{p}_{n}\right)=\prod_{i=1}^{n}\left(a_{i 1}, a_{i 2}, a_{i 3}\right)=\left(\prod_{i=1}^{n} a_{i 1}, \prod_{i=1}^{n} a_{i 2}, \prod_{i=1}^{n} a_{i 3}\right) \text {. }
$$

Similarly, if $\tilde{p}_{i}$ 's are assigned trapezoidal fuzzy numbers $\left(a_{i 1}, a_{i 2}, a_{i 3}, a_{i 4}\right), i=1,2 \ldots . n$, then

$$
\tilde{p}_{y}=\operatorname{ANDF}\left(\tilde{p}_{1}, \tilde{p}_{2}, \ldots \ldots \ldots \ldots \ldots . . . . \tilde{p}_{n}\right)=\prod_{i=1}^{n}\left(a_{i 1}, a_{i 2}, a_{i 3}, a_{i 4}\right)=\left(\prod_{i=1}^{n} a_{i 1}, \prod_{i=1}^{n} a_{i 2}, \prod_{i=1}^{n} a_{i 3}, \prod_{i=1}^{n} a_{i 4}\right)
$$

(b) Let $\tilde{p}_{i}$ 's are triangular fuzzy numbers $\left(a_{i 1}, a_{i 2}, a_{i 3}\right), i=1,2 \ldots n$, then

$$
\begin{aligned}
& \tilde{p}_{y}=\operatorname{ORF}\left(\tilde{p}_{1}, \tilde{p}_{2}, \ldots \ldots \ldots \ldots \ldots . . . . \tilde{p}_{n}\right)=1-\prod_{i=1}^{n}\left(1-\left(a_{i 1}, a_{i 2}, a_{i 3}\right)\right)=1-\prod_{i=1}^{n}\left(1-a_{i 3}, 1-a_{i 2}, 1-a_{i 1}\right) \\
& =1-\left(\prod_{i=1}^{n}\left(1-a_{i 3}\right), \prod_{i=1}^{n}\left(1-a_{i 2}\right), \prod_{i=1}^{n}\left(1-a_{i 1}\right)\right) \\
& =\left(1-\prod_{i=1}^{n}\left(1-a_{i 1}\right), 1-\prod_{i=1}^{n}\left(1-a_{i 2}\right), 1-\prod_{i=1}^{n}\left(1-a_{i 3}\right)\right)
\end{aligned}
$$

Similarly, if $\tilde{p}_{i}$ 's are trapezoidal fuzzy numbers $\left(a_{i 1}, a_{i 2}, a_{i 3}, a_{i 4}\right), i=1,2 \ldots n$, then

$$
\begin{aligned}
& \tilde{p}_{y}=\operatorname{ORF}\left(\tilde{p}_{1}, \tilde{p}_{2}, \ldots \ldots \ldots \ldots \ldots . . . \tilde{p}_{n}\right)=1-\prod_{i=1}^{n}\left(1-\left(a_{i 1}, a_{i 2}, a_{i 3}, a_{i 4}\right)\right) \\
& =\left(1-\prod_{i=1}^{n}\left(1-a_{i 1}\right), 1-\prod_{i=1}^{n}\left(1-a_{i 2}\right), 1-\prod_{i=1}^{n}\left(1-a_{i 3}\right), 1-\prod_{i=1}^{n}\left(1-a_{i 4}\right),\right.
\end{aligned}
$$

\section{Approximation of fuzzy numbers for basic events}

Using the data for the occurrence of the basic events, each basic event can be assigned an appropriate fuzzy number (triangular or trapezoidal). It is assumed that a basic event is assigned $n$ triangular fuzzy numbers $A_{i}=\left(a_{i}-c_{i} . a_{i}, a_{i}+c_{i}\right), i=1,2 \ldots n$ by $n$ different experts. We have to choose a single fuzzy number out of these $n$ triangular fuzzy numbers for any particular event that tunes with 
the judgment of all experts. Let $B=(b-d, b, b+d)$ be the number, which best fits with all experts' decision. The values of $b$ and $d$ are obtained in such a way that the fuzzy number $B$ have minimum variance with all $A_{i}$ 's. For this we find $B-A^{i}$, which is again a triangular fuzzy number. Smaller, the triangular fuzzy number $B-A^{i}$, will result in the best approximation for $B$. The height of the triangle $B-A^{i}$ cannot be reduced, since it must always be one. Therefore our measure depends on the length of base line of the triangle. For this we suppose

$$
S=\sum\left[2\left(d-c_{i}\right)\right]^{2}
$$

where $S$ denotes the sum of squares of deviations. Then $S$ will achieve its minimum if

$$
d=\frac{1}{n} \sum_{i=1}^{n} c_{i} .
$$

Also if we want to determine the parameter $b$, we consider

$$
\mathrm{D}=\max _{1 \leq i \leq n}\left|b-a_{i}\right|,
$$

where $\mathrm{D}$ denotes the absolute deviation, then $\mathrm{D}$ will be minimum for $b=\frac{\min _{1 \leq i \leq n} a_{i}+\max _{1 \leq i \leq n} a_{i}}{2}$.

This approach can be extended to approximate trapezoidal fuzzy numbers too. Let $A_{i}=\left(a_{i}-c_{i}, a_{i}, a_{i}{ }^{\prime}, a_{i}{ }^{\prime}+c_{\mathrm{i}}\right) i=1,2 \ldots . n$. are $n$ trapezoidal fuzzy numbers used to represent the failure possibility of basic events. A trapezoidal fuzzy number $B=\left(b-d, b, b^{\prime}, b^{\prime}+d\right)$ will be the fuzzy number, which has minimum variance with all $n$ fuzzy numbers. The parameters $b, b^{\prime}, d$ and $d^{\prime}$ are obtained as:

$$
d=\frac{1}{n} \sum_{i=1}^{n} c_{i}, b=\frac{\min _{1 \leq i \leq n} a_{i}^{\prime}+\max _{1 \leq i \leq n} a_{i}^{\prime}}{2} \text { and } b^{\prime}=\frac{\min _{1 \leq i \leq n} a_{i}+\max _{1 \leq i \leq n} a_{i}}{2}
$$

\section{Fuzzy importance}

In FTA we have observed that each basic event play different role in the occurrence of top event, which infers that the basic events are of different importance. Thus a critical analysis of the importance of different basic events may help in making a proper sequence of their importance. On improving the reliability of the event having greater importance, one can improve the reliability of the system. The fuzzy importance of any event is always calculated in the form of fuzzy importance index (FII). This FII may be evaluated by ranking fuzzy numbers $\left(P_{T}-P_{T i}\right)$ for $i=1,2,3 \ldots n$. Here $P_{T}$ and $P_{T_{i}}$ denote the possibility of absolute occurrence of top event and the possibility of occurrence of top event in absence of basic event $i$ respectively. In our analysis we have used less

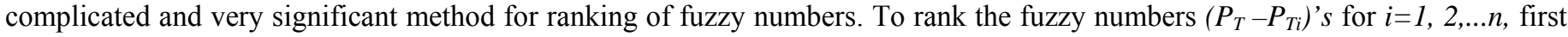
of all we have to find MAX $\left(P_{T}-P_{T i}\right), i=1,2 \ldots . n$, where the MAX operator on fuzzy numbers is defined as below.

$$
\operatorname{MAX}\left(A_{1}, A_{2}, A_{3}, \ldots \ldots \ldots . . . A_{n}\right)(z)=\sup _{z=\max \left(x_{1}, x_{2}, x_{3}-\cdots\right.}\left[A_{1}\left(x_{1}\right), A_{2}\left(x_{2}\right), A_{3}\left(x_{3}\right), \ldots \ldots \ldots \ldots A_{n}\left(x_{n}\right)\right]
$$

where $A_{1}, A_{2}, A_{3}, \ldots \ldots \ldots A_{n}$ are different fuzzy numbers

Taking the MAX of given fuzzy numbers, we try to get the distance of all these fuzzy numbers from their MAX with the help of Hamming distance formula $d_{H}(A, B)=\int_{[0,1]}|A(x)-B(x)| d x$ between two fuzzy numbers A and B.

The distance of these fuzzy numbers $P_{T}-P_{T_{i}}$ for $i=1,2 \ldots n$ from their MAX decides the rank of fuzzy numbers $P_{T}-P_{T_{i}}$. Smaller the distance of fuzzy number $P_{T}-P_{T_{1}}$ from $\operatorname{MAX}\left(P_{T}-P_{T_{i}}\right), i=1,2 \ldots n$, in comparison to distance of $P_{T}-P_{T_{2}}$ from MAX $\left(P_{T}-P_{T_{i}}\right)$ implies that fuzzy number $P_{T}-P_{T_{1}}$ is greater than $P_{T}-P_{T_{2}}$. It concludes that the fuzzy importance index (FII) may be defined in form of distance of $P_{T}$ from $P_{T i}$ i.e.

$$
F I I(i)=\frac{1}{1+\text { Dis tan ce of fuzzy number }\left(P_{T}-P_{T_{i}}\right) \text { from their MAX }}
$$

\section{Fault tree of Nuclear Power Plant}

Radiation release is assumed to be a hazard and treated as the top event in fault tree analysis. The radiation release may be caused due to the occurrence of some events, and these events may again occur due to some other events as shown in Figure 1 


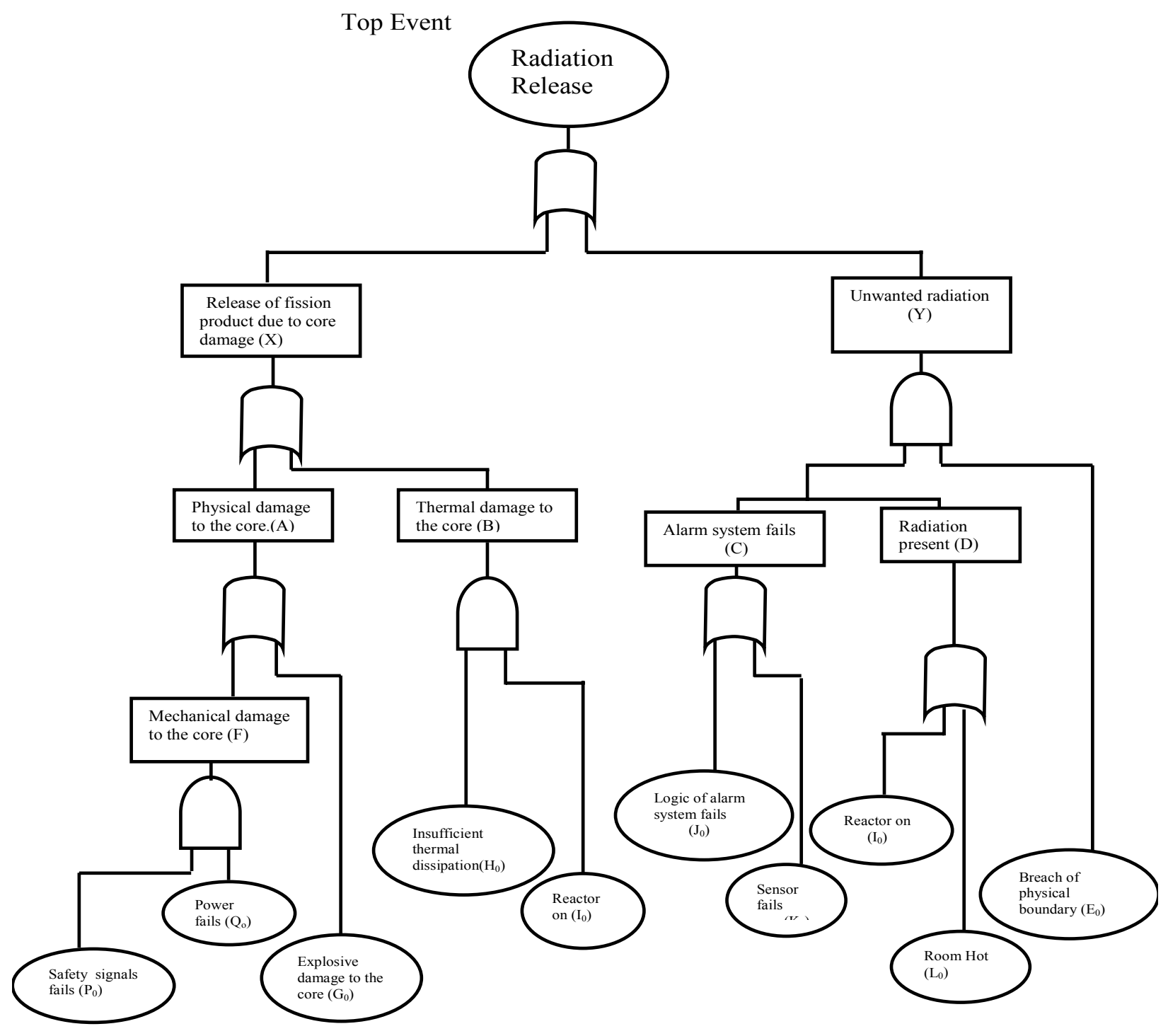

Figure 1. Fault tree of Nuclear Power Plant

The Boolean expression corresponding to this fault tree is given below:

$R R=X \cup Y, X=A \cup B, Y=C \cap D \cap E_{0}, A=F \cup G_{0}, F=P_{0} \cap Q_{0}, B=H_{0} \cap I_{0}, C=J_{0} \cup K_{0}, D=I_{0} \cup L_{0}$, where $R R$ denotes radiation release, $X=$ release of fission product due to core damage, $Y=$ unwanted radiation, A= physical damage to the core, $B=$ thermal damage to the core, $C=$ alarm system fails, $D=$ radiation present, $\mathrm{E}_{0}=$ breach of physical boundary, $F=$ mechanical damage to the core, $G_{0}=$ explosive damage to the core, $P_{0}=$ safety signal fails, $Q_{0}=$ power fails, $H_{0}$ insufficient thermal dissipation, $I_{0}=$ reactor on, $J_{0}=$ logic of alarm system fails, $K_{0}=$ sensor fails and $L_{0}=$ room hot. On replacing the Boolean operators with fuzzy logic operators FNOT, ORF and ANF, we get the possibility of the top event in form of a fuzzy number. It is also assumed that each basic event is fuzzzified by assigning three fuzzy numbers to each basic event following the decision of three experts. The different fuzzy numbers assigned to these basic events are listed in Table 1 and 2. 
Table 1. Triangular Fuzzy Numbers (Basic Events)

\begin{tabular}{|c|c|c|c|}
\hline \multicolumn{4}{|c|}{ Event $E_{0}$} \\
\hline \multicolumn{4}{|l|}{ Expert } \\
\hline 1 & 0.008 & 0.01 & 0.012 \\
\hline 2 & 0.026 & 0.03 & 0.034 \\
\hline 3 & 0.02 & 0.025 & 0.03 \\
\hline \multicolumn{4}{|c|}{ Event $\mathbf{G}_{0}$} \\
\hline Expert & $a_{1}$ & $A_{2}$ & $a_{3}$ \\
\hline 1 & 0.032 & 0.042 & 0.052 \\
\hline 2 & 0.044 & 0.05 & 0.056 \\
\hline 3 & 0.034 & 0.04 & 0.046 \\
\hline \multicolumn{4}{|c|}{ Event $P_{0}$} \\
\hline Expert & $a_{1}$ & $A_{2}$ & $a_{3}$ \\
\hline 1 & 0.075 & 0.08 & 0.085 \\
\hline 2 & 0.046 & 0.05 & 0.054 \\
\hline 3 & 0.065 & 0.07 & 0.075 \\
\hline \multicolumn{4}{|c|}{ Event $\mathbf{J}_{\mathbf{0}}$} \\
\hline Expert & $\overline{a_{1}}$ & $A_{2}$ & $a_{3}$ \\
\hline 1 & 0.042 & 0.05 & 0.058 \\
\hline 2 & 0.055 & 0.06 & 0.065 \\
\hline 3 & 0.038 & 0.045 & 0.052 \\
\hline \multicolumn{4}{|c|}{ Event $L_{0}$} \\
\hline Expert & $a_{1}$ & $A_{2}$ & $a_{3}$ \\
\hline 1 & 0.04 & 0.1 & 0.16 \\
\hline 2 & 0.16 & 0.25 & 0.34 \\
\hline 3 & 0.16 & 0.2 & 0.24 \\
\hline
\end{tabular}

Table 2. Trapezoidal Fuzzy Number (Basic Events)

\begin{tabular}{|c|c|c|c|c|}
\hline Event $\mathbf{Q}_{\mathbf{0}}$ \\
\hline Expert & $a_{1}$ & $a_{2}$ & $a_{3}$ & $a_{4}$ \\
\hline 1 & 0.072 & 0.08 & 0.085 & 0.093 \\
\hline 2 & 0.055 & 0.06 & 0.07 & 0.075 \\
\hline 3 & 0.045 & 0.05 & 0.055 & 0.06 \\
\hline Event $\mathbf{H}_{\mathbf{0}}$ \\
\hline Expert & $a_{1}$ & $a_{2}$ & $a_{3}$ & $a_{4}$ \\
\hline 1 & 0.012 & 0.02 & 0.024 & 0.032 \\
\hline 2 & 0.02 & 0.03 & 0.036 & 0.046 \\
\hline 3 & 0.034 & 0.04 & 0.042 & 0.048 \\
\hline Event $\mathbf{I}_{\mathbf{0}}$ & \multicolumn{5}{|l|}{} \\
\hline Expert & $a_{1}$ & $a_{2}$ & $a_{3}$ & $a_{4}$ \\
\hline 1 & 0.07 & 0.1 & 0.12 & 0.15 \\
\hline 2 & 0.18 & 0.2 & 0.21 & 0.23 \\
\hline 3 & 0.23 & 0.25 & 0.27 & 0.29 \\
\hline Event $\mathbf{K}_{\mathbf{0}}$ & \multicolumn{5}{|l|}{} \\
\hline Expert & $a_{1}$ & $a_{2}$ & $a_{3}$ & $a_{4}$ \\
\hline 1 & 0.12 & 0.15 & 0.16 & 0.19 \\
\hline 2 & 0.16 & 0.2 & 0.22 & 0.26 \\
\hline 3 & 0.28 & 0.3 & 0.33 & 0.35 \\
\hline
\end{tabular}

Using the approximation method discussed earlier, a single fuzzy number is obtained by which suits with all the three experts' decision for each basic event. The fuzzy numbers thus obtained for each basic event are listed in Table 3 and Table 4 .

Table 3. Approximated Triangular Fuzzy Numbers (Basic Events)

\begin{tabular}{|c|c|c|c|}
\hline Basic Event & $a_{1}$ & $a_{2}$ & $a_{3}$ \\
\hline $\mathrm{E}_{0}$ & .0163 & .02 & .0237 \\
\hline $\mathrm{G}_{0}$ & .0380 & .045 & .0520 \\
\hline $\mathrm{P}_{0}$ & .0703 & .075 & .0797 \\
\hline $\mathrm{J}_{0}$ & .045 & .052 & .059 \\
\hline $\mathrm{L}_{0}$ & .112 & .175 & .238 \\
\hline
\end{tabular}

Table 4. Approximated Trapezoidal Fuzzy Numbers (Basic Events)

\begin{tabular}{|c|l|l|l|l|}
\hline Basic Event & $a_{1}$ & $a_{2}$ & $a_{3}$ & \multicolumn{1}{|c|}{$a_{4}$} \\
\hline $\mathrm{Q}_{0}$ & .059 & .065 & .07 & .076 \\
\hline $\mathrm{H}_{0}$ & .022 & .030 & .033 & .041 \\
\hline $\mathrm{I}_{0}$ & .152 & .175 & .195 & .218 \\
\hline $\mathrm{K}_{0}$ & .195 & .225 & .245 & .275 \\
\hline
\end{tabular}

\section{FII of basic events in Nuclear Power Plant}

The fuzzy importance of each basic event can be obtained in the form of fuzzy importance index (FII) for all basic events. We calculate the possibility of top event RR using fuzzy operators and possibilities of basic events. The possibility of top event is resulted as trapezoidal fuzzy number $(.046, .056, .057, .069)$ given by the following expression. 


$$
P_{T}= \begin{cases}\frac{x-.046}{.01} & \text { if } .046 \leq x \leq .056 \\ \frac{1.069-x}{.012} & \text { if } .056 \leq x \leq .057 \\ \text { if } .057 \leq x \leq .069\end{cases}
$$

Here $P_{T i}$ 's for different events $i=E_{0}, G_{0}, P_{0}, J_{0}, L_{0}, Q_{0}, H_{0}, I_{0}$ and $K_{0}$ obtained as triangular and trapezoidal fuzzy numbers are listed in Table 5.

Table 5. Possibility of Top Event in absence of different basic events

\begin{tabular}{|c|c|}
\hline Event $(i)$ & $\begin{array}{c}\text { Possibility of top event in absence of event } i \\
\left(P_{T i}\right)\end{array}$ \\
\hline$E_{0}$ & $(.045, .054, .055, .066)$ \\
\hline$G_{0}$ & $(.008, .012, .013, .018)$ \\
\hline$P_{0}$ & $(.042, .052, .053, .063)$ \\
\hline$J_{0}$ & $(.046, .056, .057, .069)$ \\
\hline$L_{0}$ & $(.046, .055, .056, .068)$ \\
\hline$Q_{0}$ & $(.042, .052, .053, .063)$ \\
\hline$H_{0}$ & $(.043, .052, .061)$ \\
\hline$I_{0}$ & $(.042, .051, .060)$ \\
\hline$K_{0}$ & $(.045, .055, .056, .067)$ \\
\hline
\end{tabular}

First we evaluate $P_{T}-P_{T_{i}}$ and then MAX of these fuzzy events for $i=1,2 \ldots n$,. The distance of the fuzzy numbers $P_{T}-P_{T_{i}}$ from their MAX is obtained by using Hamming distance formula. Fuzzy importance index (FII) is thus obtained by the following expression.

$$
\text { FII }(i)=\frac{1}{1+\text { Dis } \tan \text { ce of fuzzy number }\left(P_{T}-P_{T_{i}}\right) \text { from their MAX }}
$$

The values of FII are obtained for all the basic events and listed in Table 6 in descending order of their importance.

Table 6. Fuzzy Importance Index of basic events

\begin{tabular}{|l|l|l|l|l|l|l|l|l|l|l|}
\hline Event $(i)$ & $G_{0}$ & $I_{0}$ & $E_{0}$ & $Q_{0}$ & $P_{0}$ & $L_{0}$ & $K_{0}$ & $H_{0}$ & $J_{0}$ \\
\hline FII $(i)$ & 1 & .9629 & .9611 & .9611 & .9611 & .9606 & .9606 & .9533 & .9367 \\
\hline
\end{tabular}

\section{Conclusion}

The techniques developed in this paper are demonstrated by taking the example of a nuclear power plant, and the following conclusions are drawn:

- All basic events $E_{0}, Q_{0}, I_{0}$ etc are assigned triangular/ trapezoidal fuzzy numbers by different experts under prescribed condition. Using technique developed in this paper, a single fuzzy number for each basic event is obtained, that tunes fine with all experts' judgments. In many situations, where the failure possibility of different basic events is collected from different experts under various operating conditions. It is more useful to adopt this realistic approach to get a single fuzzy number for this purpose.

- Applying the method developed in our study for the importance of basic events, the fuzzy importance index (FII) of basic events is calculated. The basic events are listed in Table 6, in accordance with the descending order of their FII. It is observed that the basic event $G_{o}$ is of higher sensitivity (greater importance) in comparison to other succeeding events.

- Taking note of FII of basic events listed in Table 6, it is concluded that we should emphasize on basic event $G_{o}$ rather than other succeeding events $I_{o}, E_{o}$ etc. to improve the reliability of the nuclear power plant.

- By performing the techniques developed here early in design phase of various complex systems like nuclear power plant, potential deficiencies can be identified and averted to precipitate the occurrence of various basic events causing the happening of top event(radiation release).

- Further, fuzzy numbers used herein, to represent failure possibility of basic events can be replaced with intutionistic fuzzy numbers to give more general results in fault tree analysis. 


\section{References}

Antonio C.F. Guimarees and Nelson F.F. Ebeken. 1999. Fuzzy FTA: a fuzzy fault tree system for uncertainty analysis. Annals of Nuclear Energy, Vol. 26, pp. 523-532.

Furuta H. and Shiraishi N. 1984. Fuzzy importance in fault tree analysis. Fuzzy Sets and Systems, Vol. 12, pp. 205-213.

Gozaloz A. 1990. A study of ranking function approach through mean values. Fuzzy Sets and Systems, Vol. 35, pp. $29-41$.

Grogorzewek Prezemyslow and Mrowka Edyta. 2005. Trapezoidal approximation of fuzzy numbers. Fuzzy Sets and Systems, Vol.153, pp. 115-135.

Jackson P.S., Hockenbury R.W. and Yeater M.L. 1981. Uncertainty analysis of system reliability and availability assessment. Nuclear Eng. Des., Vol. 68, pp. 5-29.

Klir G.J.and Yuan Bo. 1995. Fuzzy sets and fuzzy logic, Theory and Applications. Prentice-Hall, Upper Saddle River, NJ.

Pan Z.J. and Tau Ya-Chuan. 1988. Variance importance of system components by Monte-Carlo, IEEE Trans. Reliability, Vol. 37, 421-423.

Pandey D. and Tyagi Sanjay Kumar. 2009. Failure mode screening using fuzzy set theory. International Mathematical Forum, Vol. 4, No. 13-16, pp. $779-794$.

Pandey D. and Tyagi Sanjay Kumar. 2007. Profust Reliability of a gracefully degradable system. Fuzzy Sets and Systems, Vol. 158 , pp. 794- 803 .

Singer D. 1990. A fuzzy set approach to fault tree analysis. Fuzzy Sets and Systems 34, pp.145-155.

Suresh P.V., Babar A.K. and Venkat Raj V. 1996. Uncertainty in fault tree analysis: A fuzzy approach. Fuzzy Sets and Systems, Vol. 83, pp.135-141.

Tanaka H., Fan L.T., Lai F.S. and Toguchi K. 1983. Fault-tree analysis by fuzzy probability. IEEE Trans. Reliability, Vol. 32, pp. 453-457.

Tsajimura Yashiro and Gen Mitsuo. 1994. Fuzzy fault tree and its Importance analysis. Proceedings of $16^{\text {th }}$ 1CC \& IE. pp. 301304.

Zadeh L.A. 1965. Fuzzy sets: Information and control, Vol. 8, pp. 338-353.

\section{Biographical notes}

Dr. S. K. Tyagi is currently working as Asstt Professor of Mathematics in Amity University Noida, India. He qualified National Eligibility Test (UGC-CSIR) twice, when he appeared in M.Sc. in 2000 in Institute of Advance Studies, C.C.S. University, Meerut. Dr. Tyagi has made remarkable contribution to the research in fuzzy and classical reliability and later, he was awarded his Ph.D. degree in this area of research by Institute of Advance Studies, C.C.S. University, Meerut, India. He published several research papers in various International/National journals of repute. He has reviewed research papers for IEEE Trans. on Fuzzy Sets and Systems, and Optimization (Published by Taylor and Francis). His research interests include fuzzy and classical reliability theory, fuzzy logic and its applications. He has authored five books on Mathematics for undergraduate students.

Dr. D. Pandey received his M.Sc. from Allahabad University, Allahabad and D.Phil. from Meerut University, Meerut, India. He is Professor of Mathematics in Institute of Advanced Studies, C.C.S. University, Meerut. He has been Dean Students' Welfare, Dean, Faculty of Science, C.C.S. University, Meerut. Dr Pandey has produced fourteen Ph.D and supervised eighty one M.Phil. projects. He has published more than thirty five research papers in various International/National journal of repute. His area of research is Mathematical Analysis, Operations Research, Fuzzy Sets and its Applications and Operating Systems. He is life member of Indian Science Congress, Indian Mathematical Society and International Academy of Physical Sciences..

Reena Tyagi is currently working as Senior Lecturer in R.D. Engineering College, Ghaziabad, India. She completed her B.E. in Electronics and Communication Engg(ECE) from Dr K.N. Modi Inst. of Engg. And Technology, Modinagar(Ghaziabad), India in 2000. After doing her B.E. she joined Anu Vidut, Roorkee, India as R\&D and Testing Engineer in 2001. She has worked as a Lecturer in ECE in Meerut Institute of Engg and Technology, Meerut, and B.B.D. Inst. of Technology, Ghaziabad. She is pursuing her M.Tech in Digital Communication from Uttar Pradesh Technical University, Lucknow, India. Her research interests include Digital Communication, Microprocessor, Digital Signal Processing and Fuzzy Logic.

Received May 2010

Accepted August 2010

Final acceptance in revised form September 2010 